Check for updates

Cite this: Digital Discovery, 2022, 1, 45

\section{Predicting 3D shapes, masks, and properties of materials inside transparent containers, using the TransProteus CGI dataset $\uparrow$}

\author{
Sagi Eppel, (D)*ab Haoping Xu, bc Yi Ru Wang ${ }^{\mathrm{e}}$ and Alan Aspuru-Guzik ${ }^{\star a b c d}$
}

We present TransProteus, a dataset, and methods for predicting the 3D structure, annotations and properties of materials inside transparent vessels from a single image. Manipulating materials in containers is essential in most areas of experimental chemistry and depends heavily on vision. Training computer vision to recognize specific properties and shapes demands large annotated datasets. Manually annotated datasets are limited in size and cannot accurately account for 3D structures and continuous material properties (such as transparency). This work supplies a new procedurally generated dataset consisting of 50k images of liquids and solid materials inside transparent containers. The image annotations include 3D models, material properties (color/transparency/roughness...), and segmentation masks for the vessel and its content. The synthetic (CGI) part of the dataset was procedurally generated using 13k different objects, 500 different environments (HDRI), and 1450 material textures (PBR) combined with simulated liquids and procedurally generated vessels. In addition, we supply 104 realworld images of objects inside transparent vessels with depth maps of both the vessel and its content. We demonstrate a net that uses a single image to predict the visual material properties. We propose a method that predicts 3D models from an image as an $X Y Z$ map. This allows the trained net to predict the 3D model as a map with $X Y Z$ coordinates per pixel without prior knowledge of the image source. We use this to predict 3D models of the materials in the vessel and the vessel, using a single image.
Received 23rd September 2021 Accepted 18th December 2021

DOI: $10.1039 / d 1 d d 00014 d$

rsc.li/digitaldiscovery dramatically accelerate material discovery. The modern computer vision approach relies on training neural networks using large annotated datasets with images and their properties. Manually creating such datasets is labor-intensive and restricted to simple properties like material class (liquid/solid) and region in the image (segmentation). However, 3D structures and continuous properties like color and transparency level cannot be manually annotated with reasonable accuracy. In addition, when viewing an object through a transparent surface, considerable distortion occurs to the shape, which cannot be fully accounted for by human annotators. An alternative approach is using computer-generated images (CGI). In this approach, all the properties of the materials and the 3D shape are generated by computer and therefore known with absolute precision. However, the main challenge, in this case, is creating images that are realistic and general enough to capture the complexity of the real world. Visual properties of chemical systems can be very complex. Therefore achieving such a photorealistic result is highly complicated and not always possible. An alternative approach is to make the data highly diverse with a large variety of shapes and textures. In this case, a net trained on such a dataset will be forced to learn highly generalized representations that will enable it to handle cases not seen in the dataset. The annotation includes a 3D model of both the transparent vessel and its content (Fig. 1), segmentation masks, 


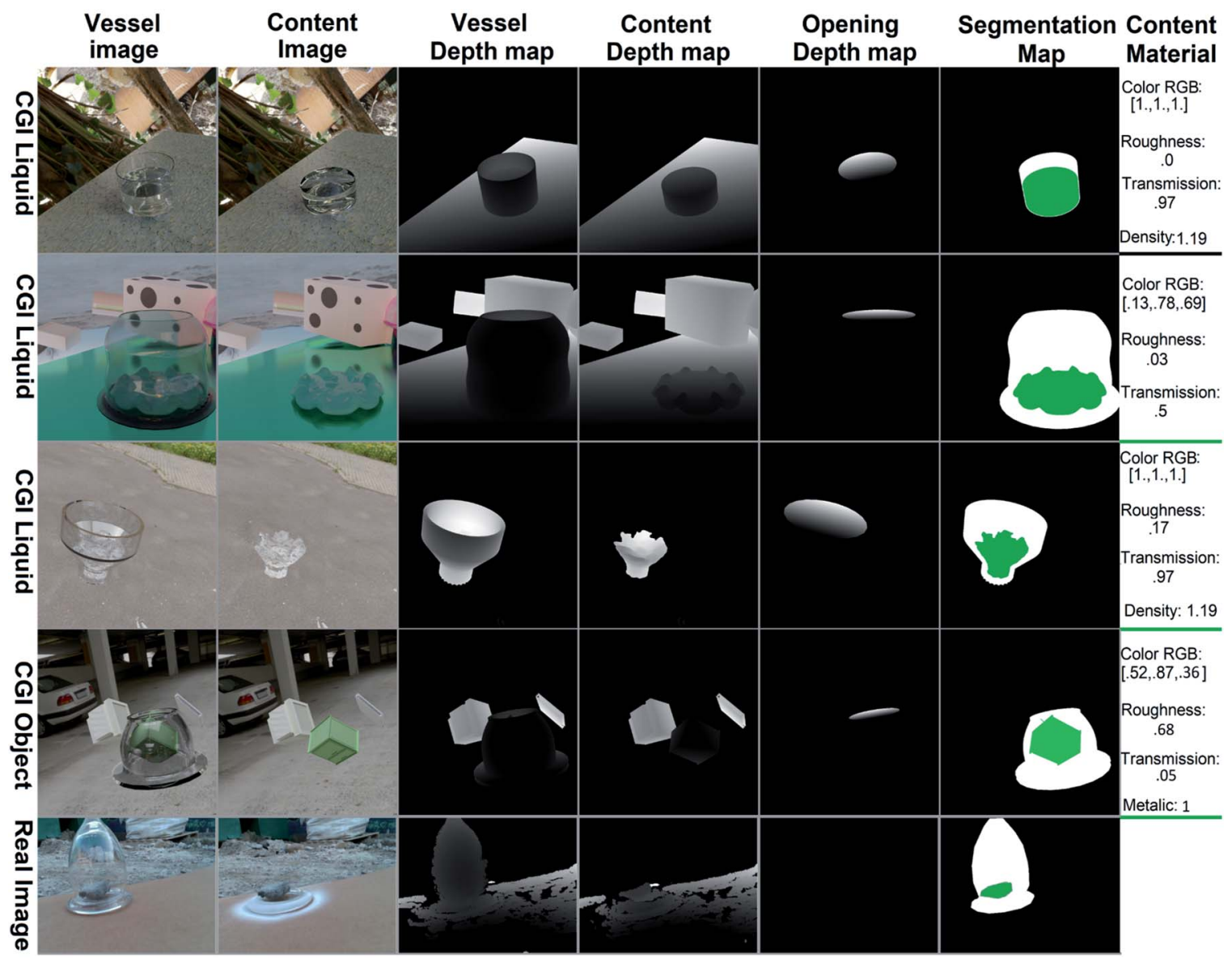

Fig. 1 The TransProteus dataset contains images of liquids and objects in transparent containers for both simulated and real pictures. Depth maps, 3D models, 2D annotations, and properties of the materials are supplied for the vessels and the materials and objects within them.

and the properties of the materials that make up the vessel and its content (color, transparency, reflectance, roughness, etc.). This work presents a new dataset focused on these tasks, which combines computer-generated images (CGI) for training and real-world photos for testing. The dataset was generated using the Blender 3D software ${ }^{4}$ with an emphasis on generality and diversity. Over 500 high-definition backgrounds (HDRI) ${ }^{5}$ were used, providing a wide variety of natural illumination and environments. In addition, over 13000 random objects ${ }^{6,7}$ were used for both the background and the vessel content. Finally, over 1400 material textures (PBRs) ${ }^{8}$ were used for the ground plane. The vessels were procedurally generated with an unlimited number of different curves, shapes, and materials. Two types of content were generated inside the container. The first type is random objects taken from the ShapeNet dataset ${ }^{6,7}$ and put inside the vessel (Fig. 3). The second content type was liquids with various properties simulated using the Blender MantaFlow ${ }^{9}$ tool with effects such as splashing, foam, and bubbles (Fig. 3). Container shapes and materials for both vessels and content were procedurally generated. Altogether, this makes the TransProteus dataset one of the most diverse synthetic datasets in terms of environment, illumination, materials, objects, and setting (Fig. 3). For comparison, the recently released ClearGrasp ${ }^{2}$ synthetic dataset for transparent objects contains 9 cad models, 33 HDRI lighting environments, and 65 materials. In addition, we created a small dataset containing 104 real-world photos with depth maps of both the vessel and its content (Fig. 1 and 3); this set was created using the RealSense depth sensor ${ }^{10}$ and used to test the net trained on the synthetic CGI dataset (Fig. 3). We also introduce a new model and training method for predicting a 3D model from a single image as an $X Y Z$ map. The prediction is independent of the camera type and image source. Previous work has already addressed scale-invariant ${ }^{11-13}$ and unknown camera parameters ${ }^{\mathbf{1 4}-16}$ when predicting depth maps from images. We expand upon this work by predicting the $X Y Z$ map instead of the depth map. ${ }^{17}$ Hence each pixel in the prediction map contains the $X, Y, Z$ coordinates of a point instead of the distance to this point (Fig. 2). This $X Y Z$ map is equivalent to the point cloud and does not depend on camera parameters. A major issue with predicting the model as a $X Y Z$ map is that the coordinates depend on the origin point, which cannot be deduced from the image. Making the $X Y Z$ prediction independent of origin (translation invariance) is achieved by using the distance between every two points in the model as the loss metric instead of the absolute $X Y Z$ coordinates (Fig. 4). The loss is simply the sum of the absolute difference $\left(L_{1}\right)$ between the normalized predicted distance and the Ground Truth (GT) distance between the same 


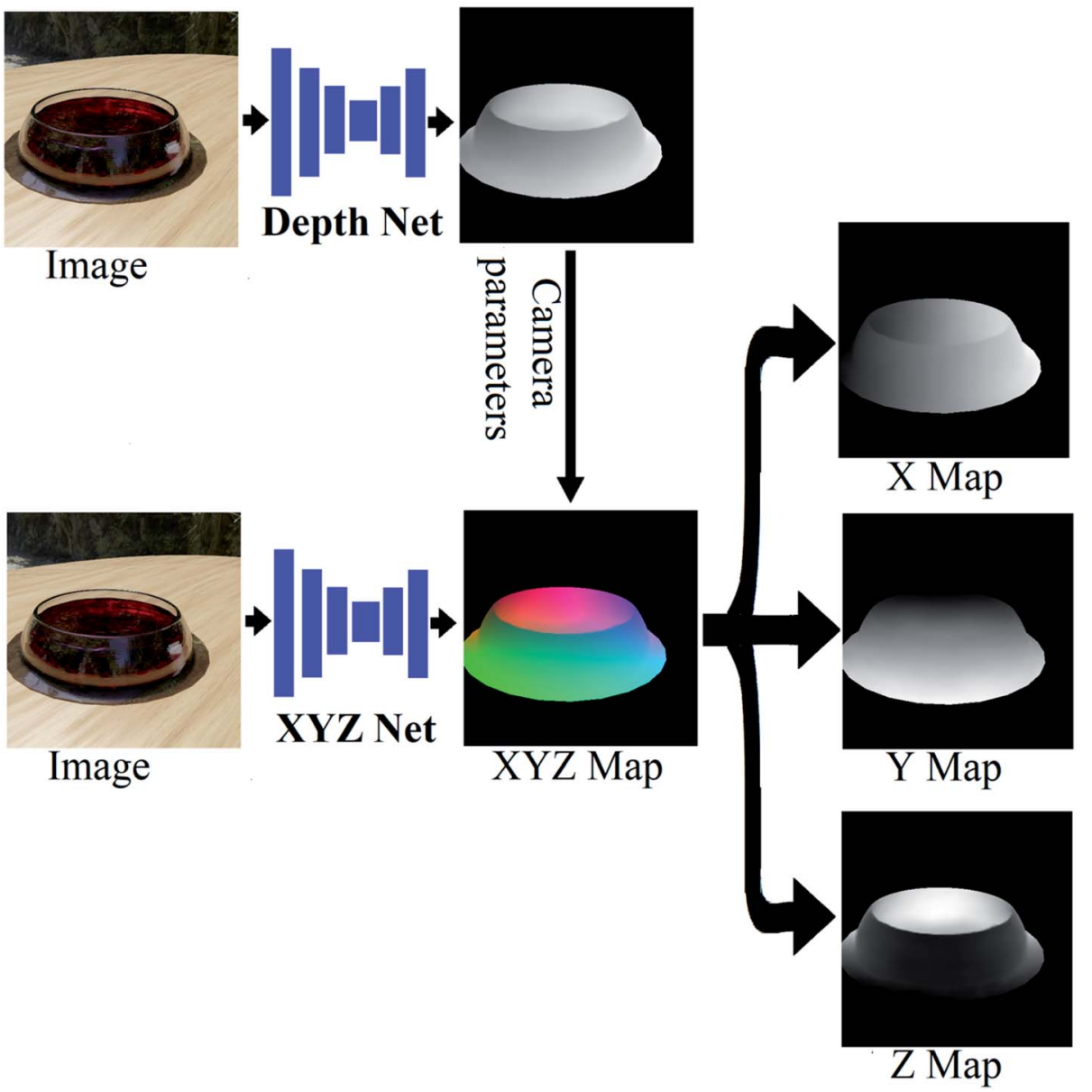

Fig. 2 Depth prediction net versus $X Y Z$ prediction net. Depth maps give the distance of every pixel from the camera. Distance is encoded as the pixel intensity/brightness. The $X Y Z$ net predicts for each pixel the $X Y Z$ coordinates as three values (three-layer map). Converting a depth map to an $X Y Z$ map is only possible using known camera parameters. The $X Y Z$ map is equivalent to a 3D model and does not require camera parameters. The $X Y Z$ map is displayed as a BGR image with the blue, green, and red values of a pixel corresponding to the pixel's $X, Y$, and $Z$ coordinates, respectively. It can be split into three $2 D$ maps for $X, Y$, and $Z$ coordinates (where the pixel intensity corresponds to the coordinate value along this axis).

pixel pairs (Fig. 4). The net used for this task is a simple, fully convolutional neural net (FCN) that outputs the $X Y Z$ map as an image with three values per pixel (Fig. 2). Another method demonstrated here is a net that receives the image and the region of the vessel in the image and predicts the properties of the materials inside the container and the vessel's material properties (Fig. 5b).

In summary, the main contributions of this work are the following:

(1) The first method and dataset for predicting 3D shapes of materials, liquids, and objects inside transparent vessels.

(2) A dataset and method to predict the properties of materials for both transparent containers and the things inside them. These properties include color, roughness, transparency, reflectance, and many other visual properties.

(3) A novel method to predict a 3D model directly from an image as an $X Y Z$ map. The prediction is independent of the image's source and camera type and can be directly converted into points cloud.
(4) A demonstration of how environments and materials repositories created for the CGI artist community can significantly increase the diversity of computer-generated synthetic datasets.

\section{Related work}

\subsection{Computer vision for materials handling in the} chemistry lab

Machine vision has been used for decades to recognize simple properties like color, turbidity, and fill level for materials in vessels for analytical chemistry. ${ }^{\mathbf{1 8 - 2 8 , 5 4 - 5 6}}$ More advanced algorithms based on methods such as graph-cut were used to segment material with unpredictable surface shapes (like solids) and multiphase materials. However, these approaches are still limited to simple conditions with controlled environments and often fail in complex real-world scenarios. Recently, methods based on deep neural nets and convolutional neural nets (CNNs) have proven significantly more effective in 
performing all of the above tasks. ${ }^{29-33}$ Semantic and instance segmentation allows one to find the region and class of each object and material phase in general conditions. However, these nets have been so far limited to 2D segmentation and cannot predict continuous materials properties or accurately account for distortions resulting from the vessel surface. ${ }^{31-33}$ On the other hand, materials manipulated in the lab can have complex 3D shapes, textures, and visual properties. Understanding this is essential for visual understanding and manipulating the experimental system.

\subsection{Predicting depth and $3 \mathrm{D}$ model from a single image}

Extracting a 3D model from photos could be achieved using a single image or multiple images covering different viewpoints. ${ }^{11-16,34-36}$ The standard depth and 3D prediction methods assume that light moves in straight lines, ${ }^{\mathbf{1 0 , 3 7}}$ an assumption that fails with transparent objects. ${ }^{1-3}$ As a result, standard methods for extracting the 3D models from images like stereo matching and structured light fail on transparent objects. ${ }^{58}$ Deep neural nets learn directly from data and therefore do not rely on any assumptions. Neural nets for extracting 3D models from images have mostly relied on using fully convolutional nets (FCNs) that predict depth maps. ${ }^{11-16}$ The value of each pixel in the depth map corresponds to the distance of this pixel from the camera (Fig. 2). This approach is assumption-free and can easily predict depth maps of transparent vessels from a single image. With known camera parameters, it is possible to convert the depth map into an $X Y Z$ map with the 3D position of each pixel in the world (Fig. 2). However, without the parameters of the camera used to take the image, it can be hard to convert the depth map into a 3D model. To solve this, several methods for extracting camera parameters from unfamiliar images were suggested ${ }^{14-16}$ However, this usually demands additional steps. Directly predicting the $X Y Z$ map (3D coordinate per pixel) can be achieved by the same methods used for the depth map but does not demand camera parameters for creating the 3D model (Fig. 2). Predicting a 3D model from a single image, as an $X Y Z$ map, was done in previous work. ${ }^{17}$ However, in this case, the loss function was based on converting the $X Y Z$ back to depth. Since this conversion depends on camera parameters, this led to the loss of the camera agnostic property of the net. Another approach for extracting 3D models from images is based on 3D convolutional nets that output a 3D grid of voxels that represent the model. This approach is more robust and better matches the problem of the $3 \mathrm{D}$ reconstruction. However, the extra dimension of the net demands a significant increase in memory and computing resources. To deal with this, most such nets work on low-resolution grids. ${ }^{59}$

\subsection{CGI and real photo dataset creation}

Deep learning approaches for computer vision are strongly reliant on training data. Generating data for specific tasks remains the main challenge in applying computer vision to new fields and improving the performance of existing fields. Creating a dataset can be done manually by collecting images and using humans for annotation. This approach mainly applies to classification ${ }^{38}$ and segmentation ${ }^{29-33}$ but can also be used for depth estimation by asking people to estimate the relative distance to two objects. ${ }^{13}$ Other methods rely on metadata or sensor data from depth sensors, LIDAR, stereo, or a structure from motion. ${ }^{34-36}$ These approaches fail on transparent objects. $^{1-3}$ Synthetic datasets use simulation and computer-generated imagery (CGI) to create the training data. ${ }^{3,39-42}$ The advantage of this approach is that it is not limited by sensors and human perception and can work in any case where the data can be simulated. However, training using this dataset often gives inferior results compared to training from real data, mainly because the simulation often misses many of the complex visual features of the real world. Such datasets have been created for autonomous driving, transparent objects, liquid dynamics, and material properties. ${ }^{3,39-42}$ However, as far as we know, no such dataset was suggested for materials and objects inside transparent containers. Another issue with existing synthetic datasets is the use of a small, limited set of objects and environments. For example, the ClearGrasp ${ }^{2}$ synthetic dataset for transparent objects contains 9 cad models, 33 HDRI lighting environments, and 65 materials. This makes nets trained on these datasets very limited in terms of the domain in which they can be used. Recently projects like Poly-Haven, ${ }^{5}$ ambientCCG, ${ }^{8}$ and ShapeNet ${ }^{6,7}$ created huge repositories for objects, environments, and material textures for the CGI artist community, with thousands of diverse and free samples. Using these can dramatically increase the diversity of synthetic datasets.

\subsection{Transparent object datasets}

Datasets for the segmentation of transparent objects in realworld images have been mostly created by manual annotation and image mating. ${ }^{43-46,57}$ The largest of these datasets is Trans $10 \mathrm{k},{ }^{45}$ with $10 \mathrm{k}$ images in which the region of the transparent object is marked. The LabPics dataset contains $8 \mathrm{k}$ images of mostly transparent vessels in labs, hospitals, and other settings. ${ }^{31,32}$ The vessel's content and transparent regions are manually annotated. For 3D and depth maps of transparent objects, there is still a limited number of datasets. The ClearGrasp dataset contains mostly simulated 3D data for transparent objects but with no content. ${ }^{3}$ This dataset also collected 3D scans of real transparent objects sprayed with opaque spray and then scanned with a RealSense depth sensor. Both approaches are used in this work as well.

\section{Dataset generation}

The goal of the TransProteus dataset is to allow the prediction of the 3D shapes, segmentation maps and properties of materials, liquids, and objects inside vessels regardless of the application. However, the large number of research fields, industrial applications, and everyday life activities for which this problem is relevant means that it's impossible to simulate all the different objects and materials that can occur within the vessel, even for a specific field like experimental chemistry. To address this, we try to make the dataset as general as possible, assuming that if 


\section{a. Simulated liquid in vessel}
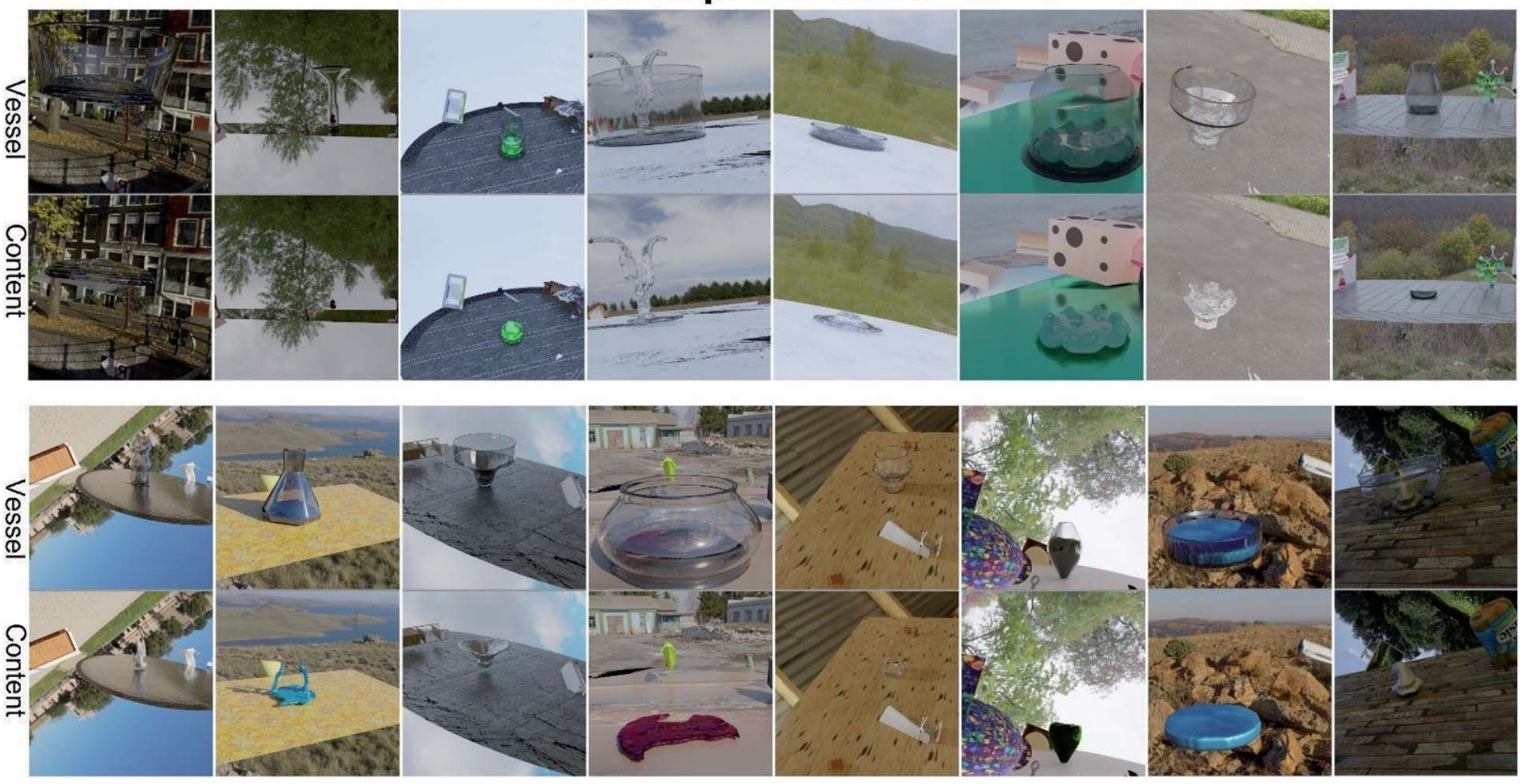

\section{b. Simulated object in vessel}
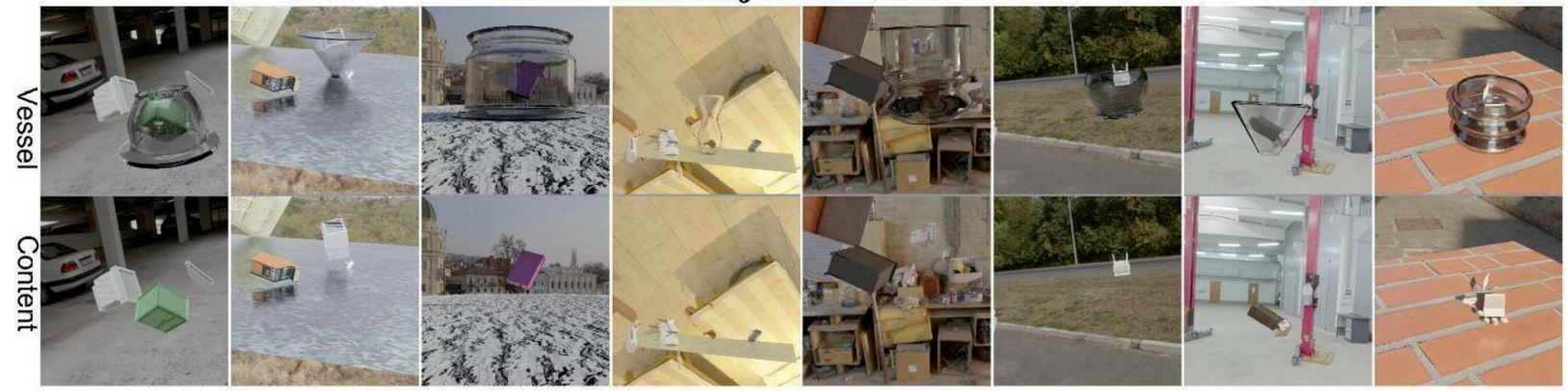

\section{c. Real-world photos}
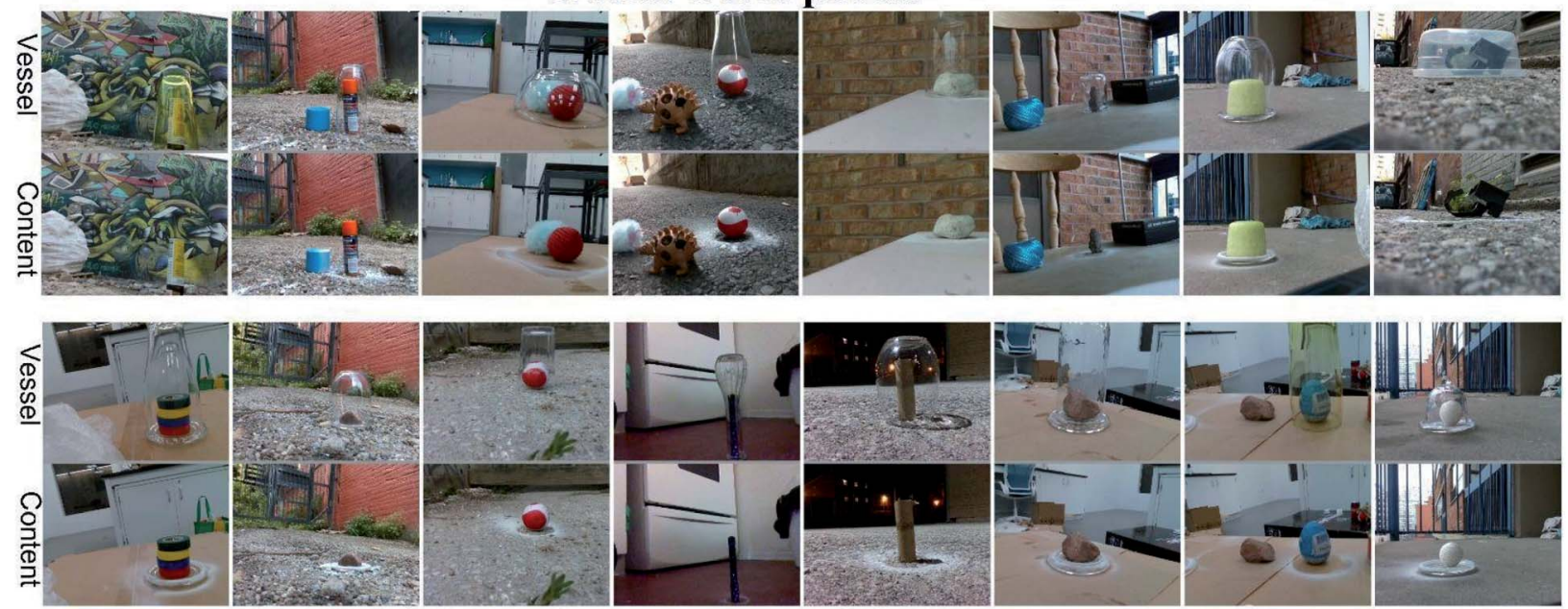

Fig. 3 Example of simulated images for liquids and objects in vessels and real-world photos of things in containers. Each line contains the same scene, with the vessel and without the vessel (exposed content). (a) CGl images of simulated liquids. (b) CGl images of objects in vessels. (c) Realworld images of objects in vessels. 
the dataset contains diverse enough examples, any network trained on this dataset will be able to generalize to new and unfamiliar systems. This means that instead of narrowing down the generated data to be as realistic as possible for a given use case, we try to make it as diverse as possible, even if many of the examples are unlikely to exist in reality. For example, the environments for both the real and simulated datasets include a large variety of backgrounds, including fields, parking lots, and many other locations that are not usually used to handle transparent containers (Fig. 3). In addition, the content of each vessel was chosen to be a random object or liquid with random properties. Many of these examples are very unlikely to appear in reality (Fig. 3). However, the variability and diversity of the dataset mean that any network that will learn to predict the shape and properties of all of these different cases will have to be highly generalized and work in almost any case. Since the goal of the dataset is to train machine learning systems, we take generality and diversity and not realism as the main focus. Code for generating the dataset is supplied in the ESI. $\dagger$

\subsection{Computer-generated environments}

The simulated dataset was generated using Blender. Creating background and illumination for the scenes was done using high dynamic range images (HDRI). These high-definition images completely surround the scene, providing background and full natural illumination from all directions. Five hundred HDRIs were downloaded from the Poly Haven project. The HDRI environments include indoor and outdoor settings in cities, nature, and other environments (Fig. 3). To further increase diversity, the HDRIs were rotated, and their intensity increased or decreased randomly for every image. To make the scene even more diverse, we use the objects from the ShapeNet dataset. We used 13k different objects from a large number of categories. Up to 10 random objects were randomly scattered in every scene, with random scale position and rotation. A ground plane was generated by creating a simple plane below the objects and assigning random physically based rendering $(\mathrm{PBR})^{47}$ materials to this plane. ${ }^{3}$ These PBR materials contain realistic complex textures, displacements, and other properties of real-world materials. About 1445 different material textures were downloaded from the ambientCG project. We note that the steps taken so far are not specific to this dataset and can be used to create a general setting and environment in any synthetic dataset. They can greatly benefit synthetic datasets that tend to use a limited set of environments and settings. Generating the synthetic data was done using scripts that create random procedurally generated scenes that are different in every image.

\subsection{Procedurally generating vessels}

Glassware shapes vary widely between different use cases. However, almost all glassware tends to have cylindrical or symmetric shapes (from a top view). Hence, we can describe every vessel's top view as a circle or other symmetric shape, and the vessel curvature (profile) is some 2D function (Fig. 3). The curvature (profile) was generated by randomly combining linear, polynomial, and sinusoidal functions to create the vessel curvature derivative, leading to a random but mostly smooth $2 \mathrm{D}$ function. This leads to a wide range of shapes that seem to cover any vessel we encounter in the real world (Fig. 3).

\subsection{Generating liquids and objects for vessel content}

The contents of the vessel were generated using one of three methods. The first approach was to take a set of random objects from the ShapeNet dataset and randomly position them inside the vessel. This is by far the most diverse method for filling the vessel but the least realistic (Fig. 3, center). The second approach was to create a random blob of liquid inside the vessel. The liquid was given a random shape, properties, and initial velocity and was simulated using the MantaFlow module of Blender. Images were captured in various steps of the simulation. This creates a wide variety of shapes associated with liquid splashing, spilling, and sticking, which covers the wide range of liquid behaviors in the real world (Fig. 3, top). The MantaFlow liquid simulation also contains tools for simulating foam, and bubbles, which were used. The final approach for content creation is to represent the static liquid as a mesh with a flat surface that fills the bottom part of the vessel (Fig. 1, top). No actual liquid simulation is needed in this case. This is the most common way liquid will appear in vessels, but it has the least diversity.

\subsection{Generating and assigning materials}

Materials for both the vessel and its contents were generated using the principled BSDF material ${ }^{48}$ shader in Blender. This tool enables the control of all the visual properties of the material, including color, transmission (transparency), metallic (reflection), IOR, roughness, luminescence, and many others. The materials generated by this tool are uniform, and unlike PBR materials, they do not have complex textures (Fig. 3). However, glass vessels and liquids tend to be very homogenous anyway. The advantage of BSDF materials over PBR materials is that all the visual properties are given as a list of numbers with constant length. ${ }^{48}$ This can be saved and later predicted by the net. For the case of objects inside the vessels, the objects are already supplied with materials and textures (from the ShapeNet dataset). Therefore, the materials of objects inside the vessels were kept as they are for $50 \%$ of cases and replaced by uniform principled BSDF materials in the remaining 50\%. For liquids and the vessel, randomly generated BSDF materials were used for all cases. It should be noted that liquid splashing, foam, and bubbles effects also influence the liquid material textures. These properties are not described by the BSDF shader and cannot be predicted using the dataset.

\subsection{Creating a real-world image dataset}

Generating real-world images and 3D scans of transparent objects and their content is challenging for two main reasons. First, standard depth sensors like LIDAR and structure light (RealSense ${ }^{\mathbf{1 0}}$ and Kinect) do not work on transparent vessels. Second, scanning the depth map of the vessel content requires a method to remove the vessel without moving its content or the sensor (because depth sensors can't penetrate the vessel surface). The problem of 3D scanning transparent vessels was solved by first taking an image of 
the vessel and then spraying it with opaque spray without moving the vessel or the sensor, similar to the method used in the ClearGrasp dataset. ${ }^{3}$ Removing the vessel without moving or changing its content is a more challenging task. To achieve this, the vessel was put upside down over an object, scanned, and then removed without moving the object or sensor (Fig. 3, bottom). This approach is clearly valid only for solid content. In addition, it relies on the ability of the nets to work with an arbitrary orientation of the vessel. The images were taken in various buildings, parking lots, and yards, with a large set of random vessels and objects (14 locations, 20 vessel types, and 25 different objects inside the vessels). The real sense D435 depth sensor was used for scanning, and AESUB blue spray was used for painting the vessels. This procedure is time-consuming. Therefore, only 104 images were collected and used for testing the dataset. The annotation of the vessel and content masks was done manually on the images of the vessel and exposed content. The RealSense ${ }^{\mathbf{1 0}}$ depth data is very noisy. The depth map was cleaned by removing points that are more than $10 \mathrm{~cm}$ from the object center. Since all the vessels used are smaller than $10 \mathrm{~cm}$, a distance of more than $10 \mathrm{~cm}$ from the center implies an error in measurement.

\section{Predicting 3D model as an $X Y Z$ map}

Predicting the 3D model as an $X Y Z$ map can easily be done using a fully convolutional neural net (FCN) that receives an image and predicts the $X Y Z$ map as a three-layer image (Fig. 2).

\subsection{Translation invariance loss}

Since the coordinates of the $X Y Z$ points have an arbitrary origin point (assuming camera parameters are unknown), it is necessary that the loss will be independent of the origin point (translation invariant). Translation invariant loss can be achieved by using the distance between points in the model as the metric instead of the point's $X, Y, Z$ coordinates (Fig. $4 \mathrm{~b}$ ). This is because the distances between two points in the model do not depend on their absolute coordinates or the origin point.

This is illustrated in Fig. 4b: assume that the vertical axis is some line in the image plane (in pixels) and the horizontal axis is the $Z$ coordinates in $\mathrm{cm}$. The predicted map is translated relative to the GT map, leading to completely different $Z$ coordinate values for GT and predicted maps, even when the shapes are similar (Fig. 4b). However, the difference in $Z$ coordinates $\left(D_{z, 1}\right)$ between two points $\left(p_{1}, p_{2}\right)$ is independent of origin. Therefore similar GT and predicted shapes should have the same $D_{z}$ value (Fig. $2 \mathrm{~b}$ ).

If $D_{z, i}^{\mathrm{GT}}=Z_{1}^{\mathrm{GT}}-Z_{2}^{\mathrm{GT}}$ is the difference between the $Z$ coordinates in pixels 1 and 2 in the GT map (Fig. 4b), and $D_{z, i}^{\mathrm{Prd}}=$ $Z_{1}^{\text {Prd }}-Z_{2}^{\text {Prd }}$ is the $Z$ difference between the same two pixels in the predicted $X Y Z$ map, then $\left|D_{z, i}^{\mathrm{Prd}}-D_{z, i}^{\mathrm{GT}}\right|$ is the translation independent error/loss.

The loss function is, therefore, the absolute mean of differences between these distances, along each of the axes and for every pair of pixels in the object:

$$
\text { Loss }=\operatorname{mean}\left(\left|D_{a, i}^{\mathrm{GT}}-D_{a, i}^{\mathrm{Prd}}\right|\right)
$$

$i \in$ all pairs of pixels inside the objects, $a \in X, Y, Z$ axes.

\subsection{Scale-invariant loss}

The above loss is translation invariant but still scale-dependent. This means that if the predicted model has the same shape but a different scale than the GT model, the error will be high. In our case, the scale of the 3D model cannot be extracted from the image. We, therefore, want the loss to be independent of the predicted model scale. This can be solved by adding a scale normalization constant to the predicted distance (Fig. 4). This basically means finding the scale difference between the predicted and GT models and rescaling the predicted model to match the GT model's scale.

Finding the scale factor $(K)$ could be done by taking the ratio between the sum absolute distances $(D)$ between every pair of points (and along each axis) in the GT and predicted $X Y Z$ maps:

$$
K=\frac{\operatorname{mean}\left(\left|D_{a, i}^{\mathrm{GT}}\right|\right)}{\operatorname{mean}\left(\left|D_{a, i}^{\mathrm{Prd}}\right|\right)}
$$

$i \in$ all pairs of pixels inside the objects, $a \in X, Y, Z$ axes.

This leads to a scale-invariant loss function:

$$
\text { Loss }=\operatorname{mean}\left(\left|D_{a, i}^{\mathrm{GT}}-K \cdot D_{a, i}^{\mathrm{Prd}}\right|\right) .
$$

Note that this was done only to positive distance ratios $\frac{D_{a, i}^{\mathrm{GT}}}{D_{a, i}^{\mathrm{Prd}}}>0$. The scale factor $(K)$ is calculated once for the entire image and multiplied by the predicted distances to match them to the GT distances. In addition, we want to avoid relative scales $(K)$ which are too big or small. This is because very large or small relative scales cause the training loss to explode or get stuck.

We, therefore, add a scale controlling term to the loss function that is used only if the scale factor $(K)$ is larger than ten or smaller than 0.1 . If $K>10$, this term is mean $(K)$, which causes the scale ratio to decrease, while if $K<0.1$, we add the term - mean $(K)$ to the loss function, which forces the prediction scale $(K)$ to increase. This extra loss element guarantees that the scale factor $(K)$ will always be in the range of 0.1-10.

\section{Predicting material properties}

The material properties are given as a list of numbers that include RGB color, transmission (transparency), roughness, metallic (reflectiveness), IOR, and others (Fig. 5b). Standard convolutional neural nets for image classification can easily be modified to predict these values by using the final output vector of the net to represent these properties (Fig. 5b). This was done for the material properties of both the vessel and its content. The vessel region was added as an input for the net by processing it using a single convolutional layer and adding the result to the first layer of the convolutional net (Fig. 5b). The training was done using standard ResNext ${ }^{49}$ training methods. 
a)

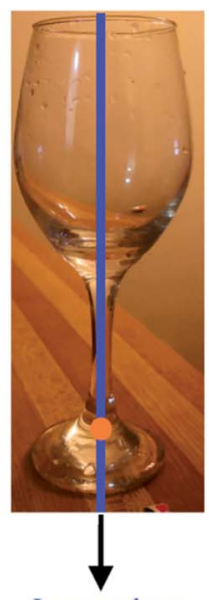

Image plane cross-section

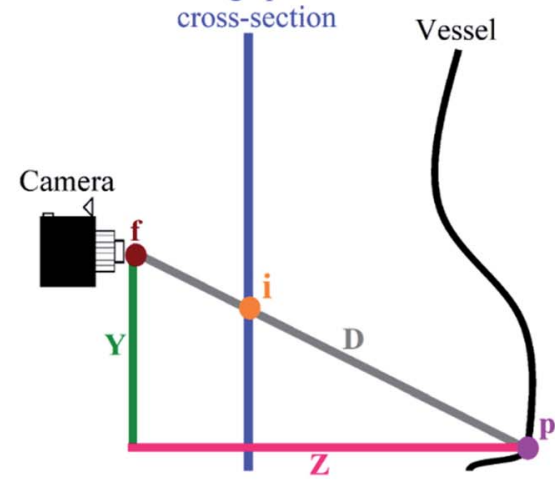

$\mathbf{f}=$ Focal point $($ Camera $)$

$\mathrm{i}=$ Pixel point

$\mathrm{p}=$ point on vessel

$\mathrm{D}=$ Distance to vessel (depth) b)

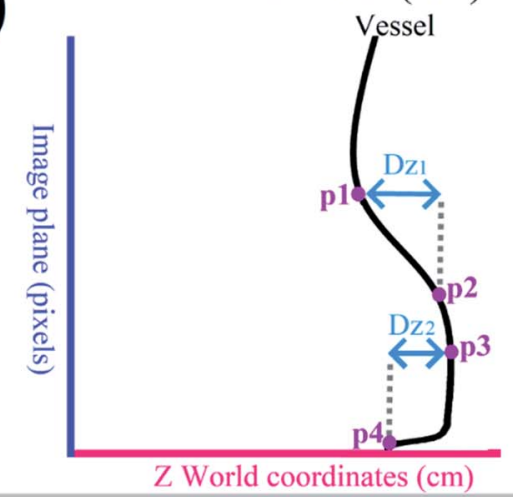

Predicted (Prd)

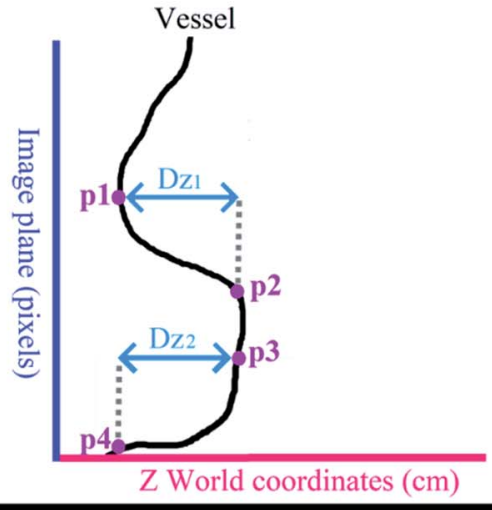

$\operatorname{Loss}=\operatorname{Mean}\left(\left|D_{\mathrm{a}, \mathrm{i}}^{\mathrm{GT}}-\mathrm{D}_{\mathrm{a}, \mathrm{i}}^{\mathrm{Prd}} \cdot \mathrm{K}\right|\right) \quad \mathrm{K}=\frac{\operatorname{Mean}\left(\left|\mathrm{D}_{\mathrm{a}, \mathrm{i}}^{\mathrm{GT}}\right|\right)}{\operatorname{Mean}\left(\left|D_{\mathrm{a}, \mathrm{i}}^{\mathrm{Prd}}\right|\right)}$

$\mathrm{a} \in \mathrm{x}, \mathrm{y}, \mathrm{z}$ coordinates

$i \in$ All pixel pairs inside the object region

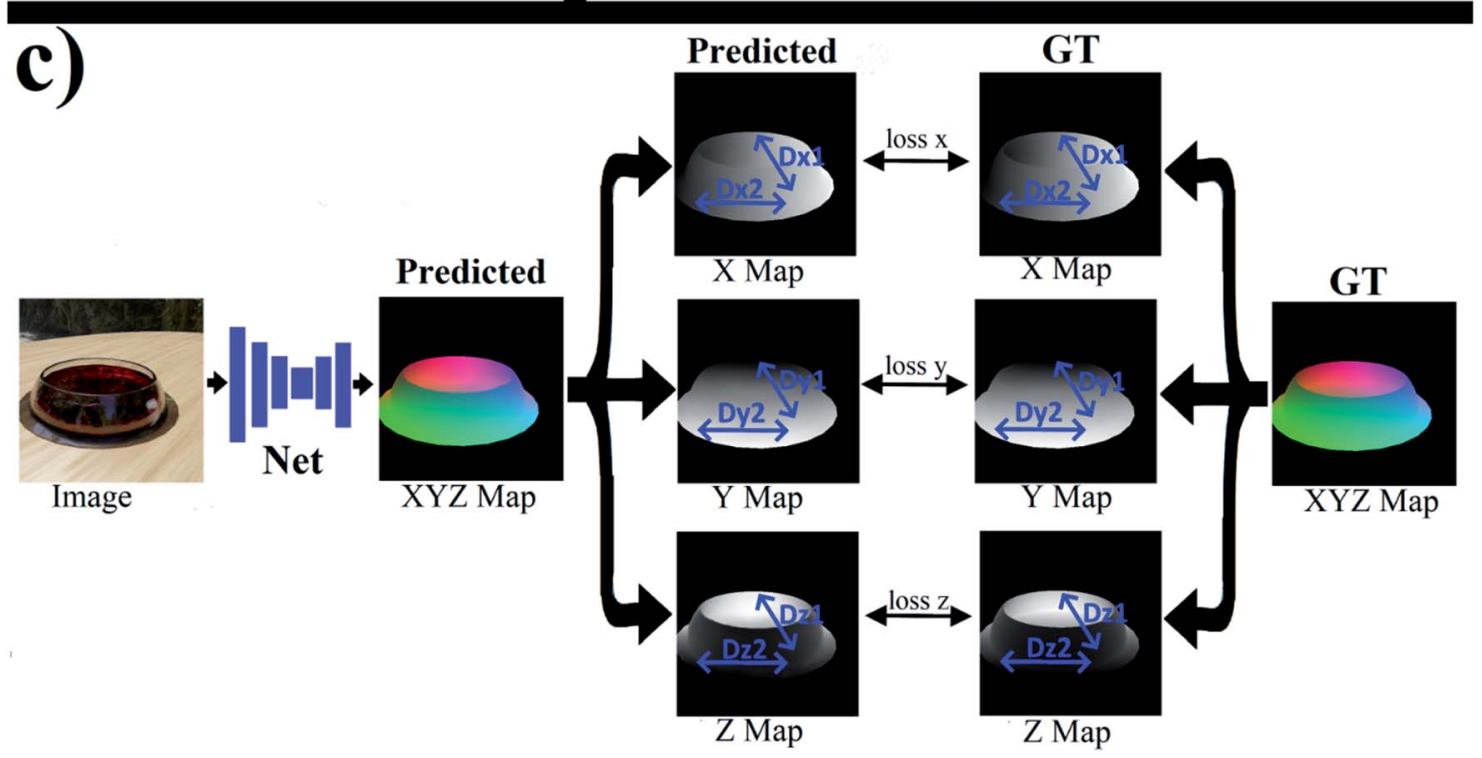

Fig. 4 Predicting 3D model as $X Y Z$ map and difference-based loss function. (a) Cross-section of the predicted map along one image column (blue line). (b) Profiles of the predicted and GT maps in the $Z$ coordinates. The predicted and GT maps' translation and scale are inconsistent with each other. Therefore, the distances between the points $\left(D_{x}, D_{y}, D_{z}\right)$ are used for the loss function instead of the absolute $x, y, z$ coordinates of the points (making the translation irrelevant). (c) Loss display on the 2D XYZ images. The distances $\left(D_{z}, D_{y}, D_{x}\right)$ refer to the difference between the two points in the $x, y$, and $z$ coordinates, respectively (and NOT to the distance in pixels between the two points on the image plane). 
a) XYZ Net

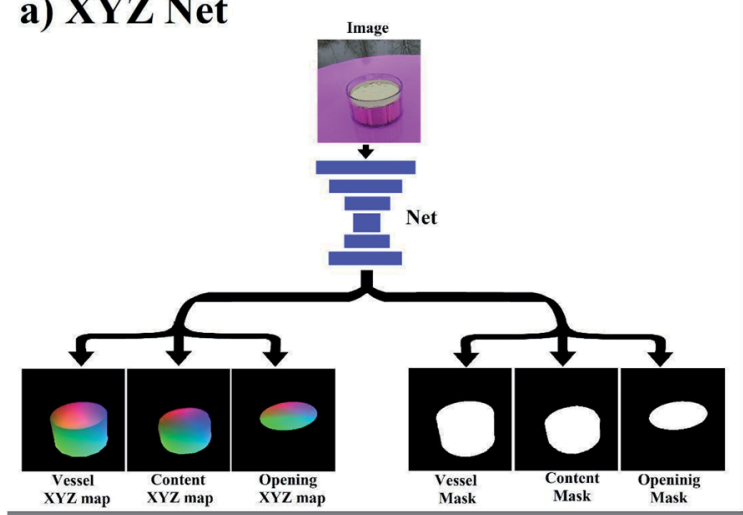

Net block

Convolutional layer

\section{b) Material properties net}

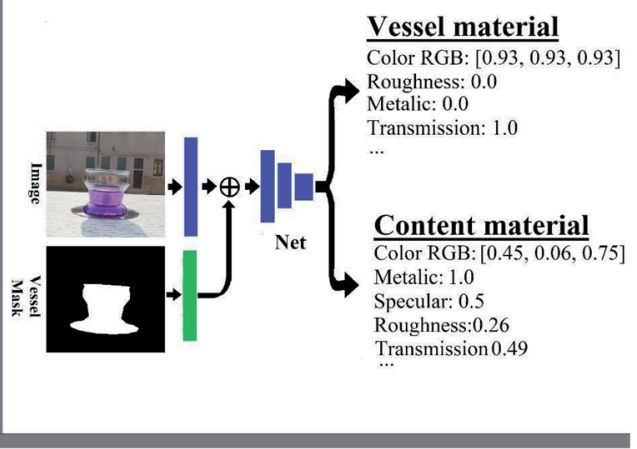

$\bigoplus$ Elementwise addition

Fig. 5 Structure of neural nets. (a) $X Y Z$ prediction net is a simple $F C N$ that produces the $X Y Z$ map as a three-layer map with $X, Y, Z$ values per pixel. The objects' masks are predicted as two-channel probability maps with two values per pixel (belongs/does not belong to the object). (b) The material property prediction net is a simple convolutional net (Resnext) that receives the image and the vessel mask (region) and predicts the properties of the vessel and content materials as a vector.

The loss function was the sum of the absolute difference between the predicted and GT vectors.

\section{Training with additional datasets}

To improve results on real-world images, we also use vessel mask and content regions from the LabPics dataset as additional training data for vessel and content mask prediction. These were applied in $30 \%$ of the training steps (the $X Y Z$ map loss was set to zero in this case).

\section{Evaluation}

\subsection{D model evaluation}

Evaluation of the $X Y Z$ map prediction was done by modifying three standard metrics. The mean absolute error (MAE) ${ }^{\mathbf{1 1}}$ takes the mean Euclidean distance between the predicted and GT points for every pixel belonging to the object:

$$
\mathrm{MAE}=\operatorname{mean}\left(D\left(p_{i}^{\mathrm{GT}}, p_{i}^{\mathrm{Prd}}\right)\right)
$$

where $D$ is the Euclidean distance between the points $p_{i}^{\text {GT }}, p_{i}^{\text {Prd }}$ for the same pixel $(i)$ on the GT and predicted $X Y Z$ maps and $i \in$ all the pixels in the object region.

In addition, since scales are arbitrary, the MAE value has little meaning. We, therefore, normalized the MAE by dividing it by one of two values: the mean absolute deviation (MAD) is the mean distance between points in the GT object and the GT object's center, was used as the first normalization factor:

$$
\mathrm{MAD}=\operatorname{mean}\left(D\left(p_{i}^{\mathrm{GT}}, c^{\mathrm{GT}}\right)\right)
$$

where, $c^{\mathrm{GT}}$ is the center (average) of the GT points.

In addition, we use the maximum distance between two points in the GT object (MaxDst) as a second normalization factor for the MAE:

$$
\text { MaxDst }=\max \left(D\left(p_{i}^{\mathrm{GT}}, p_{j}^{\mathrm{GT}}\right)\right)
$$

$i, j \in$ all pixels in the object region.

An additional metric is the standard $R$-squared, which uses the sum of the squared Euclidean distances between the predicted and GT points for every pixel divided by the sum of the mean squared distance between the GT points and the GT object's center:

$$
\begin{aligned}
R^{2} & =1-\frac{\mathrm{RSS}}{\mathrm{TSS}}, \text { with } \mathrm{RSS}=\sum_{i}^{n} D\left(p_{i}^{\mathrm{GT}}, p_{i}^{\mathrm{Prd}}\right)^{2}, \text { and TSS } \\
& =\sum_{i}^{n} D\left(p_{i}^{\mathrm{GT}}, c^{\mathrm{GT}}\right)^{2}
\end{aligned}
$$

An additional metric is the Chamfer distance, ${ }^{53}$ which is calculated by finding for each point in the GT object the closest predicted point (Euclidean distance) and finding the mean of this distance:

$$
d_{\mathrm{cd}}\left(S_{1}, S_{2}\right)=\sum_{x \in S_{1}}\left\|\min _{y \in S_{2}}(D(x, y))\right\|+\sum_{x \in S_{2}}\left\|\min _{y \in S_{1}}(D(x, y))\right\|,
$$

where $S_{1}, S_{2} \subseteq R^{3}$ for the predicted and GT point clouds, respectively.

Note that the Chamfer distance ignores the position of the points on the image grid and uses only their $X Y Z$ position. As in the case of the MAE, the distance was normalized by both the mean deviation (MAD) and the maximum deviation (MaxDst) between points on the GT object.

\subsection{Effect of scale and translation on error}

The predicted $X Y Z$ map is scaled and translated to match the GT object (Section 4). This normalization removes two types of errors (scale and translation) and leaves only the errors resulting from the object shape. However, the scaling and translation factors are found using the vessel object and apply to the content object. Therefore, the content prediction still contains 
both scaling and translation errors. In principle, this is not a problem since we expect the scale and translation factors of the vessel and content to be the same (otherwise, the content will be out of proportion and position to the vessel containing it). However, it's also interesting to isolate the shape error of the content. To achieve this, we also calculate the scale and translation factors using the content object (instead of the vessel) and use these to scale and translate the predicted content object to match the GT content.

\subsection{Evaluating material properties prediction}

Since every property of the material is represented as one or more numbers, the prediction accuracy was evaluated using the mean absolute error (MAE) between the predicted and GT material properties. This is simply the mean absolute difference between the predicted and GT values. Note that all the predicted properties have a value ranging between 0 and 1 . Hence, the MAE could also be considered as the difference between the predicted and real values by percentage. It was not possible to collect these material properties from real images; therefore, the evaluation is based on the CGI images only.

\subsection{Evaluation of $2 \mathrm{D}$ instance and semantic segmentation}

For evaluating the segmentation of the regions belonging to the content, vessel, and opening in the image, we choose the standard IOU metrics. The intersection over union (IOU) is the main metric used to evaluate semantic segmentation and is calculated separately for each object. The intersection is the sum of the pixels that belong to the object according to both the net prediction and the dataset ground truth (GT), while the union is the sum of pixels that belong to the object based on either the net prediction or the GT. The IOU is the intersection divided by the union. The recall is the intersection divided by the sum of all pixels belonging to the object according to the GT annotation, while precision is the intersection divided by the sum of all pixels belonging to the object based on the net prediction.

\section{Results}

\subsection{Results for 3D model prediction}

The results for the 3D model $X Y Z$ prediction appear in Tables 1 and 2 and Fig. 6 . It can be seen from Table 1 that the net achieves good accuracy for predicting the 3D shape of the vessel for both real and simulated pictures. This is true even when the net

Table 1 Results for $X Y Z$ net on vessel 3D model prediction (Section 7.1) ${ }^{a}$

\begin{tabular}{|c|c|c|c|c|c|c|}
\hline Test data & Net/training & MAE/MAD & MAE/MaxDist & Chamfer/MAD & Chamfer/MaxDist & $R^{2}$ \\
\hline Real images & XYZ net (with LabPics) & $12.1 \%$ & $2.5 \%$ & $15.9 \%$ & $3.3 \%$ & 0.96 \\
\hline Object vessels (RealSense) & $X Y Z$ net (only TransProteus) & $14.6 \%$ & $2.9 \%$ & $17.4 \%$ & $3.5 \%$ & 0.94 \\
\hline Simulated object in a vessel & $X Y Z$ net (with LabPics) & $8.0 \%$ & $1.7 \%$ & $9.5 \%$ & $1.7 \%$ & 0.97 \\
\hline Vessel opening plane & $X Y Z$ net (with LabPics) & $11.7 \%$ & $1.5 \%$ & $9.8 \%$ & $1.2 \%$ & 0.95 \\
\hline
\end{tabular}

${ }^{a}$ MAE: mean absolute Euclidean distance between GT and predicted points on the same pixel pairs. MAD: mean absolute deviation of the GT. Mean Euclidean distance between points on the GT object and the GT object center ( $X, Y, Z$ average). MaxDist: maximal Euclidean distance between any two points on the GT object. Chamfer distance: mean Euclidean distance between each point on the GT object and closest predicted point, plus mean Euclidean distance between each point on the predicted object and the closest GT point.

Table 2 Results for $X Y Z$ net on content 3D model prediction (Section 7.1) for predicted content scale/translation normalized by vessel object and normalized to match GT content object ${ }^{a}$

\begin{tabular}{|c|c|c|c|c|c|c|c|c|c|c|c|}
\hline Test data & Net/training & \multicolumn{5}{|c|}{ Content $X Y Z$ (normalized to content scale) } & \multicolumn{5}{|c|}{ Content (normalized to vessel scale) } \\
\hline Real images & $\begin{array}{l}X Y Z \text { net (with } \\
\text { LabPics) }\end{array}$ & $21.9 \%$ & $2.8 \%$ & $30.1 \%$ & $3.9 \%$ & 0.89 & $52.7 \%$ & $2.9 \%$ & $74.8 \%$ & $6.5 \%$ & 0.27 \\
\hline $\begin{array}{l}\text { Object vessels } \\
\text { (RealSense) }\end{array}$ & $\begin{array}{l}X Y Z \text { net (only } \\
\text { TransProteus) }\end{array}$ & $22.8 \%$ & $3.0 \%$ & $30.8 \%$ & $4.0 \%$ & 0.87 & $54.0 \%$ & $6.6 \%$ & $76.0 \%$ & $9.4 \%$ & 0.36 \\
\hline $\begin{array}{l}\text { Simulated object in } \\
\text { a vessel }\end{array}$ & $\begin{array}{l}X Y Z \text { net (with } \\
\text { LabPics) }\end{array}$ & $36.0 \%$ & $3.6 \%$ & $36.0 \%$ & $3.6 \%$ & 0.73 & $38.5 \%$ & $3.6 \%$ & $45.2 \%$ & $4.3 \%$ & 0.62 \\
\hline
\end{tabular}


is trained only on CGI images and tested on real photos (Table 1). Training with additional real images (LabPics dataset) gives only a minor advantage in this case. For content prediction in real-world images (Table 2), the net gives good accuracy for predicting the 3D shape of the object in the vessel (Section 7.2). However, when the scale and translation are
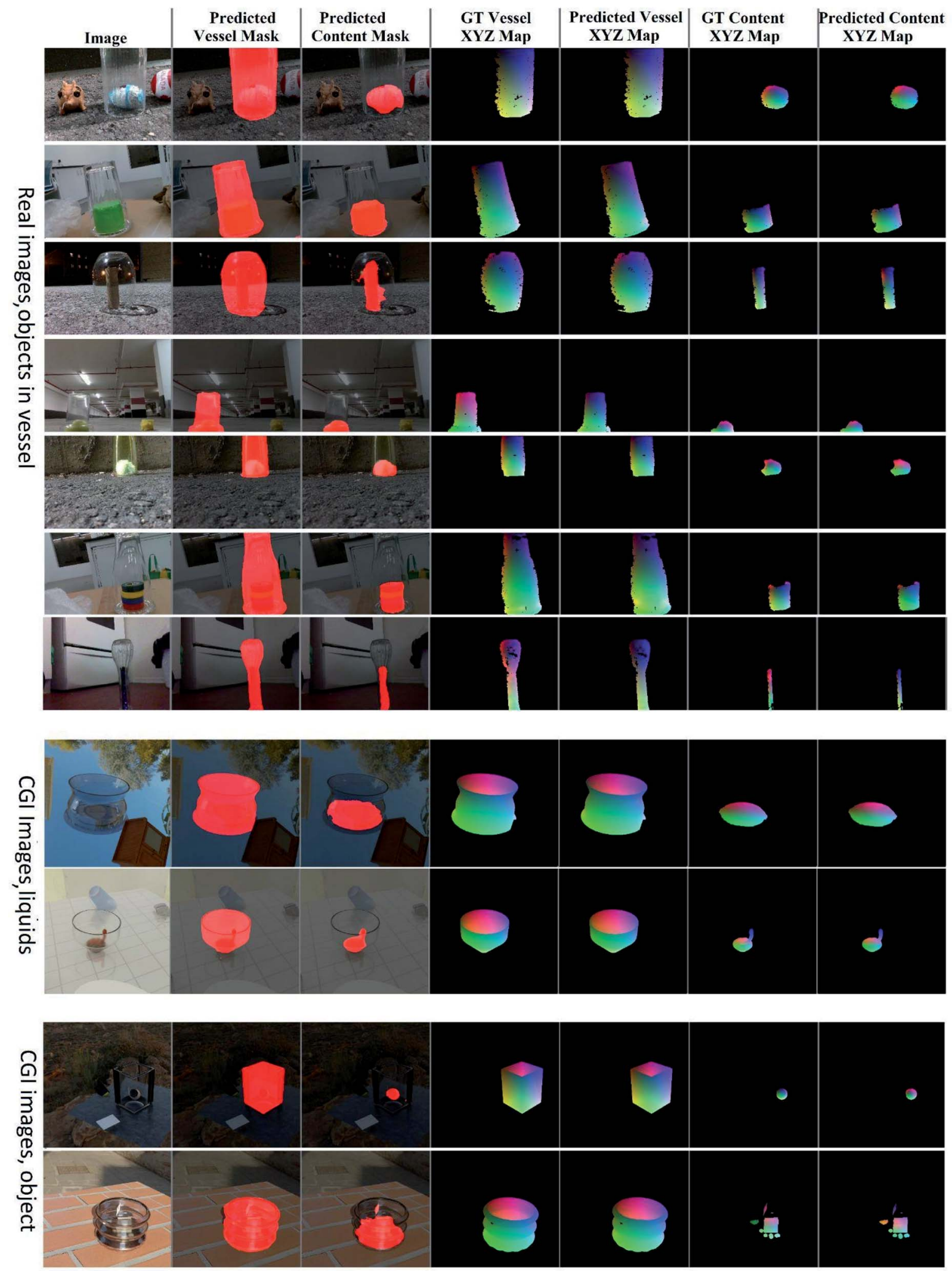

Fig. 6 Results of the neural net prediction for $X Y Z$ map and segmentation map for simulated and real images. 
Table 3 Material properties prediction mean absolute error (MAE) for content and vessel surface (Section 7.3)

\begin{tabular}{llll}
\hline & \multicolumn{2}{l}{ Mean absolute error (MAE) } & \\
\cline { 2 - 4 } Property & Content liquid & Content object & Vessel surface \\
\hline Transmission/transparency & $2.1 \%$ & $5.6 \%$ & $0.5 \%$ \\
Color (RGB) & $10.7 \%$ & $4.1 \%$ & $4.6 \%$ \\
Metallic/reflectiveness & $2.9 \%$ & $4.8 \%$ & $0.7 \%$ \\
Roughness & $5.3 \%$ & $3.9 \%$ & $0.6 \%$
\end{tabular}

Table 4 Results of semantic segmentation on real images and simulated images (Section 7.4) for net training on only TransProteus, and for a net trained on TransProteus combined with the LabPics dataset (real photos)

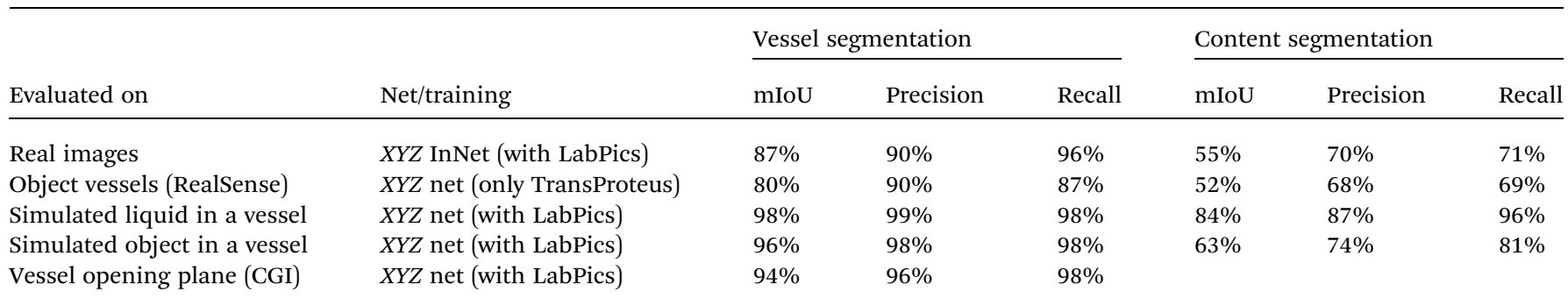

included in the error, the accuracy of the prediction is significantly lower (Table 2). This can be explained by the fact that the exact position and scale of the object in the vessel are often hard to determine from the image (Fig. 3, 6), while the object's shape is usually clear. The net achieves good accuracy in predicting the shape of liquids inside the vessel even when including translation and scale errors (Table 2, Fig. 6). This can be explained by the fact that liquids tend to either completely fill the bottom portion of the vessel or stick to the vessel surface (Fig. 3). In both cases, the position (translation) inside the vessel is clear. For simulated objects inside vessels, the net achieves medium accuracy for shape prediction (Table 3, Fig. 6). However, adding translation and scale errors did not significantly affect the accuracy (Table 3 ). This can be attributed to the significant variance in the shape of the object used (Fig. 3). The vessel opening plane was predicted with high accuracy (Fig. 1, Table 1), similar to the vessel shape predictions. This makes sense, given that the vessel opening can be viewed as the top part of the vessel.

8.1.1. Evaluation on real photos of liquids in vessels. The main limitation of the evaluation set is the lack of real images of vessels containing liquids. Since it's not possible to remove the vessel while keeping the liquid in the same place, it's not actually possible to get a 3D scan of the liquid inside the vessel. However, it is possible to run the net on real images of vessels containing liquids and qualitatively evaluate the results. We supply several videos of models generated from real images of liquids as ESI $\dagger$ in this URL: https://zenodo.org/record/5697212. It can be seen that the net results are visually similar even for complex cases like a liquid in the state of pouring. We also note that predicting the 3D shape of objects inside transparent vessels is much harder than for liquid, which, when static, has a very limited range of shapes. This can be seen by comparing the net result on the simulated images of liquids inside vessels which gives far better accuracy compared to simulated objects inside vessels (Table 2).
Hence, the result of the net on real images of objects inside transparent vessels can be viewed as the lower bound for accuracy that can be expected for real images containing liquid.

\subsection{Results for material properties prediction}

The results of material properties prediction are given in Table 3. It can be seen that the net achieves good accuracy with a mean absolute error of less than $10 \%$ for all properties. The vessel material is also predicted with high accuracy (Table 3), but in that case, the range of the material properties of the vessel surface is narrow, making the prediction relatively easy. For the content material properties, the variance in the generated materials is high in all properties (color, transparency/ transmission, roughness, and metallic/reflectiveness). Also, the difference in illumination and vessel surface reflection is quite significant (Fig. 3). Even so, the net achieves good accuracy for all properties, implying that it learns to compensate for background illumination and the vessel surface.

\subsection{Segmentation results}

The results for the segmentation of vessels and content are given in Table 4 and Fig. 6 . The net predicts the vessel region with high accuracy (IOU $>80 \%$ ) and the content region with medium accuracy (IOU $>50 \%$ ) for real and simulated images (Fig. 6). Training the net using a combination of the virtual

Table 5 Comparison of method for $X Y Z$ map prediction vs. depth map prediction on the SUN3D dataset

\begin{tabular}{lllllll}
\hline & MAE & & & \multicolumn{2}{l}{ Chamfer } & \\
\cline { 2 - 3 } Net/training & MAD & MaxDist & & MAD & MaxDist & $R^{2}$ \\
\hline Depth net & $12.7 \%$ & $3.8 \%$ & & $11.2 \%$ & $3.6 \%$ & 0.920 \\
XYZ net & $15.0 \%$ & $4.5 \%$ & & $12.5 \%$ & $3.9 \%$ & 0.904
\end{tabular}


TransProteus images and the real images of the LabPics dataset gave a $7 \%$ improvement for the vessel $2 \mathrm{D}$ segmentation in the real photos but only a $3 \%$ improvement for the vessel content for the same photos (Table 4). It should be noted that the task of the $2 \mathrm{D}$ segmentation of transparent containers and their content is also covered by the LabPics dataset. However, the LabPics dataset predicts the shape of the content as it is viewed through the vessel's transparent surface (distorted shape). On the other hand, the TransProteus dataset predicts the undistorted content shape, which is the region of the object as it would be viewed if the vessel was not in the way (Fig. 1).

\subsection{Comparing results of $X Y Z$ map prediction $v s$. depth map prediction}

Predicting depth map is the main method used to extract 3D model for image, ${ }^{11}$ and while this approach demands camera parameters in order to extract 3D model from the image, it is interesting to compare it to $X Y Z$ map on a standard dataset. We use the SUN3D dataset, ${ }^{61}$ which contains RGBD images and depth scans of various indoor scenes. Unlike the Transproteus, the camera parameters of this dataset are the same between the test set and train set. The net that was used was the same as the $X Y Z$ net, with the final layer replaced with depth map prediction. The loss function was the standard scale-invariant depth prediction. ${ }^{11}$ The output of the depth net was transformed into $X Y Z$ coordinates using the known camera parameters. The results were compared to that of the net train on predicting the $X Y Z$ map in the same dataset. Both the $X Y Z$ and depth prediction nets were trained in the same way, with only the final layer and loss function differing. The results are given in Table 5. It can be seen that the depth map prediction gives about $10-20 \%$ better results, suggesting that for known camera parameters predicting the model as a depth map is still preferable over predicting the 3D model as an $X Y Z$ map.

\section{Conclusion}

This work demonstrates the first method and dataset for predicting the 3D shape and properties of materials, liquids, and objects inside transparent containers. The nets achieve good results for simulated and real-world images, but considerable challenges remain in terms of prediction accuracy and evaluation methods. We also introduce a simple method to predict a 3D model from an image using a neural net that is independent of camera parameters and can work with images from unknown sources. For the creation of the dataset, we use existing methods for rendering and simulation. However, we combine this with large textures repositories and HDRI repositories used by CGI artists. These repositories are relatively unutilized in the machine learning community, and using them dramatically increases the diversity and generality of the dataset, making it one of the most general synthetic datasets in terms of environments, materials, and objects. As a result, the net trained on this synthetic data alone achieves good results on $3 \mathrm{D}$ and $2 \mathrm{D}$ shape prediction for real-world complex images. As a result, the net trained on this synthetic data alone achieves good results on $3 \mathrm{D}$ and $2 \mathrm{D}$ shape prediction for real-world complex images, as well as material properties of CGI images. To conclude, the TransProteus dataset solves two of the main problems in the visual understanding of chemistry. It supplies an unlimited amount of training data and allows the annotation of properties that cannot be accurately quantified or described by humans. A main limitation of the dataset is the fact that current CGI tools cannot capture the visual complexity of chemical systems. Future challenges include expanding the dataset for multiphase materials systems (phase separating liquids, suspension), simulating more complex chemical systems, and increasing the prediction accuracy.

\section{Appendix}

\subsection{Implementation details}

The $X Y Z$ net was implemented using a standard FCN (DeepLab) ${ }^{50,51}$ with Resnet101 (ref. 49) encoder, ASPP dilated convolution decoder, and three layers of skip connection + upsampling (UNet hourglass structure ${ }^{52}$ ). The final layer of the net was split into predicting the $X Y Z$ maps of the vessel, the vessel content, and the vessel opening (Fig. 5a). Each of these maps includes three layers which give the $X, Y, Z$ coordinates for each pixel (Fig. 5a). The loss for each map was calculated as described in Section 4, only for the region of the object as given by the GT mask. In addition, the regions of the vessel opening and vessel content in the image were predicted as $2 \mathrm{D}$ masks (Fig. 5a). Each mask was predicted as a two-layer probability mask (pixel belongs/does not belong to the object). The loss for each mask was calculated using the standard per pixel crossentropy function. The net was trained on a single RTX 3090 GPU. The training and net structure were the same as for a standard FCN for semantic segmentation. The PyTorch implementation and trained models have been made available. Image augmentation included resizing, centered cropping, blurring, decoloring, and adding white noise but NOT mirror reflecting and rotation. The net was trained once for all content types, with $40 \%$ of the training steps using simulated objects as the vessel content, $40 \%$ using simulated liquids as the vessel content, and the remaining $20 \%$ using liquids with a flat surface (Section 3.3).

\subsection{Hierarchical loss for vessel and content 3D shape}

We want to predict the $X Y Z$ map for the vessel and its content as well as the vessel opening surface (Fig. 5a). They can be considered as 3 different overlapping objects. Predicting $X Y Z$ maps for these three objects independently using the loss function in Section 4.2 will lead to different scales and translations for each object. To solve this, we calculate the scale factor $(K)$ for the vessel and use it for the vessel content and opening. However, since the loss function is translation invariant, this will lead to different translations for different objects. To solve this, we subtract the $X Y Z$ map of the content and the vessel in every pixel in which the vessel and the content overlap. The $L_{1}$ distance of this property (between prediction 
and GT) is used as a consistency loss that promises a similar translation for all objects.

$\begin{aligned} \text { Translation consistency loss } & =\operatorname{mean}\left(\mid p_{a, i}^{\text {vessel,GT }}-p_{a, i}^{\text {content,GT }}+\right. \\ p_{a, i}^{\text {vessel,Prd }}- & \left.p_{a, i}^{\text {content,Prd }} \mid\right)\end{aligned}$ where $p_{a, i}$ is the coordinate of the point in pixel $i$ on axis $a . i \in$ all the pixels in the image where the vessel and content overlap. $a \in X, Y, Z$ axes. Prd and GT are the predicted and ground truth, respectively. Vessel, content, refers to the type of object the point in pixel $i$ belongs to (note that only pixels where the content object and vessel object overlap are used for this loss).

\subsection{Efficient calculation of distances and loss using dilated convolution}

The difference between the $X Y Z$ coordinates for two pixels in the $X Y Z$ map can be easily calculated using a convolutional operation with a filter $[1,-1]$. Calculating the distance between faraway pixels can be done using dilated convolution with the distance as dilation $[1,0,0, \ldots,-1]$. This enables the calculation of both the loss and the scale constant as convolutional operations, which significantly improves the running time.

\subsection{Generating images, annotation, and depth maps using Blender}

Images were created using Blender cycles, a ray-tracing rendering tool (Fig. 1 and 3). Depth maps and normal maps for vessels and content were generated using Blender rendering tools and saved as .exr files. Depth maps for content were generated by simply removing the vessel from the scene, leaving its interior exposed (Fig. 1 and 3). The region of the vessel and content objects we're given by the vessel and content masks (Fig. 1). These masks were generated by comparing the scene depth maps with and without the object and marking the regions that changed (by subtracting the depth maps). In addition, the vessel opening plane was saved as a depth map and mask (Fig. 1). This is not an actual object but identifying it is important for many applications. In order to diversify scenes, the camera position and rotation, and parameters were changed randomly for each scene and are supplied in the dataset.

\section{Data availability}

Subset of the dataset can be found in this URL:

https://zenodo.org/record/5508261\#.YUoZL3tE1H4

The full dataset (over $100 \mathrm{~GB}$ ), can be download from either of these URLS:

https://e.pcloud.link/publink/show?

code $=\mathrm{kZfx} 55 Z x 1 \mathrm{GOrl} 4 \mathrm{aUwXDrifAHUPSt7QUAIfV}$

https://icedrive.net/1/6cZbP5dkNG

Code for generating the dataset can be find in these URLS: https://github.com/sagieppel/

Procedural_Annotated_Images_Generation_Liquid_Transpere nt_Vessel
https://zenodo.org/record/5511208\#.YUodY3tE1H4

Code for predicting 3D model as $X Y Z$ from anq image can be found in these URLS:

https://github.com/sagieppel/Predicting-3D-shape-of-liquidand-objects-inside-transparent-vessels-as-XYZ-map-from-asingle-image

https://zenodo.org/record/5511198\#.YUoamHtE1H4

Code for predicting material properties from an image can be found in these URLs:

https://github.com/sagieppel/Predicting-Material-

properties-of-objects-and-liquids-inside-transparent-vesselsfrom-image

https://zenodo.org/record/5511206\#.YUocHntE1H4

\section{Author contributions}

Sagi Eppel, Haoping $\mathrm{Xu}$ and $\mathrm{Yi} \mathrm{Ru}$ Wang wrote the code for generating the dataset. Nets training and evaluation was done by Sagi Eppel.

\section{Conflicts of interest}

There are no conflicts to declare.

\section{Acknowledgements}

We acknowledge the Defense Advanced Research Projects Agency (DARPA) under the Accelerated Molecular Discovery Program under Cooperative Agreement HR00111920027, dated August 1, 2019. The content of the information presented in this work does not necessarily reflect the position or the policy of the Government. A. A.-G. thanks Anders G. Frøseth for his generous support.

\section{References}

1 Z. Li, Y.-Y. Yeh, and M. Chandraker, Through the looking glass: neural 3D reconstruction of transparent shapes, Proceedings of the IEEE/CVF Conference on Computer Vision and Pattern Recognition, 2020.

2 O. Wiles and A. Zisserman, Learning to predict $3 \mathrm{~d}$ surfaces of sculptures from single and multiple views, Int. J. Comput. Vis., 2019, 127(11), 1780-1800.

3 S. Sajjan, et al., Clear grasp: 3d shape estimation of transparent objects for manipulation, 2020 IEEE International Conference on Robotics and Automation (ICRA), IEEE, 2020.

4 Blender 2.93, 2021, https://www.blender.org/.

5 Poly Haven, 2021, https://polyhaven.com/.

6 A. X. Chang, et al., Shapenet: An information-rich 3d model repository, 2015, arXiv preprint arXiv:1512.03012.

7 Shapenet. https://shapenet.org/.

8 AmbientCG, 2021, https://ambientcg.com/.

9 MantaFlow. 2021, http://mantaflow.com/.

10 L. Keselman, et al., Intel RealSense stereoscopic depth cameras, Proceedings of the IEEE Conference on Computer Vision and Pattern Recognition Workshops, 2017. 
11 D. Eigen, C. Puhrsch, and R. Fergus, Depth map prediction from a single image using a multi-scale deep network, 2014, arXiv preprint arXiv:1406.2283.

12 Q. Li, et al., Deep Learning based Monocular Depth Prediction: Datasets, Methods and Applications, 2020, arXiv preprint arXiv:2011.04123.

$13 \mathrm{~K}$. Xian, et al., Structure-guided ranking loss for single image depth prediction, Proceedings of the IEEE/CVF Conference on Computer Vision and Pattern Recognition, 2020.

$14 \mathrm{~W}$. Yin, et al., Learning to recover $3 \mathrm{~d}$ scene shape from a single image, Proceedings of the IEEE/CVF Conference on Computer Vision and Pattern Recognition, 2021.

$15 \mathrm{~W}$. Yin, et al., Diversedepth: Affine-invariant depth prediction using diverse data, 2020, arXiv preprint arXiv:2002.00569.

16 Y. Xiong, et al., Camera focal length from distances in a single image, Vis. Comput., 2021, 1-13.

17 C.-H. Lin, C. Kong, and S. Lucey, Learning efficient point cloud generation for dense $3 \mathrm{~d}$ object reconstruction, Proceedings of the AAAI Conference on Artificial Intelligence, 2018, vol. 32, no. 1 .

18 P. Shiri, et al., Automated solubility screening platform using computer vision, Iscience, 2021, 24(3), 102176.

19 B. Laÿ, Image processing: a key to success in industrial applications, Mathematical Morphology and Its Applications to Image Processing, Springer, Dordrecht, 1994, pp. 341-352.

$20 \mathrm{H}$. S. Teo, A feasibility study of a machine vision based pour control system, The University of Texas at El Paso, 1991.

21 M. Kurahashi, H. Tomikawa, and Y. Doi, Method and device for detecting average liquid level in a bottle, US Pat., 4,733,095, 22 Mar. 1988.

22 A. S. Malowany, R. Kurz, and M. Abdol-Reza, Detecting glass fibers using computer vision, Canadian Information Processing Society Graphics Interface 1986: Proceedings 5 p(SEE N 87-12175 03-61), 1986.

23 J. M. Munson, Liquid Level Sensor, Proc. Iowa Acad. Sci., 1961, 68(1), 502-503, https://scholarworks.uni.edu/pias/ vol68/iss1/68/.

24 P. Extance and G. D. Pitt, Intelligent turbidity monitoring, Meas. Control, 1984, 17(9), 343-349.

$25 \mathrm{~J}$. O. Harris, The Assembly and Use of an Automatic Turbidity Recording Device, Appl. Microbiol., 1958, 6(4), 266-268.

26 J. C. H. Chung, M. Litt, and G. Leininger, A computer vision system for automated corn seed purity analysis, Proceedings of the 3rd international conference on Industrial and engineering applications of artificial intelligence and expert systems, 1990, vol. 1.

27 B. W. Watson, et al., A simple turbidity cell for continuously monitoring the growth of bacteria, Phys. Med. Biol., 1969, 14(4), 555.

28 S. Eppel, Tracing liquid level and material boundaries in transparent vessels using the graph cut computer vision approach, 2016, arXiv preprint arXiv:1602.00177.

29 A. Xompero, et al., Multi-modal estimation of the properties of containers and their content: survey and evaluation, 2021, arXiv preprint arXiv:2107.12719.
30 R. Mottaghi, et al., See the glass half full: Reasoning about liquid containers, their volume and content, Proceedings of the IEEE International Conference on Computer Vision, 2017.

31 S. Eppel, et al., Computer vision for recognition of materials and vessels in chemistry lab settings and the vector-LabPics data set, ACS Cent. Sci., 2020, 6(10), 1743-1752.

32 S. Eppel, H. Xu, and A. Aspuru-Guzik, Computer vision for liquid samples in hospitals and medical labs using hierarchical image segmentation and relations prediction, 2021, arXiv preprint arXiv:2105.01456.

33 S. Eppel, Setting an attention region for convolutional neural networks using region selective features, for recognition of materials within glass vessels, 2017, arXiv preprint arXiv:1708.08711.

$34 \mathrm{~W}$. Chen, et al., Single-image depth perception in the wild, Adv. Neural Inf. Process. Syst., 2016, 29, 730-738.

35 W. Chen, S. Qian, and D. Jia, Learning single-image depth from videos using quality assessment networks, Proceedings of the IEEE/CVF Conference on Computer Vision and Pattern Recognition, 2019.

36 Z. Li, and N. Snavely, Megadepth: Learning single-view depth prediction from internet photos, Proceedings of the IEEE Conference on Computer Vision and Pattern Recognition, 2018.

37 U. R. Dhond, and J. K. Aggarwal., Structure from stereoa review, IEEE transactions on systems, man, and cybernetics 19.6, 1989, pp. 1489-1510.

38 A. Krizhevsky, I. Sutskever and G. E. Hinton, Imagenet classification with deep convolutional neural networks, Adv. Neural Inf. Process. Syst., 2012, 25, 1097-1105.

39 A. Gaidon, et al., Virtual worlds as proxy for multi-object tracking analysis, Proceedings of the IEEE conference on computer vision and pattern recognition, 2016.

40 Y. Tian, et al., Training and testing object detectors with virtual images, IEEE/CAA J. Autom. Sin., 2018, 5(2), 539-546.

$41 \mathrm{G}$. Ros, et al., The synthia dataset: A large collection of synthetic images for semantic segmentation of urban scenes, Proceedings of the IEEE conference on computer vision and pattern recognition, 2016.

42 C. Schenck and D. Fox, Perceiving and reasoning about liquids using fully convolutional networks, Int. J. Robot. Res., 2018, 37(4-5), 452-471.

43 J. Zhang, et al., Trans4Trans: Efficient transformer for transparent object segmentation to help visually impaired people navigate in the real world, 2021, arXiv preprint arXiv:2107.03172.

44 M. P. Khaing, and M. Masayuki, Transparent object detection using convolutional neural network, International Conference on Big Data Analysis and Deep Learning Applications, Springer, Singapore, 2018.

45 E. Xie, et al., Segmenting transparent objects in the wild, Computer Vision-ECCV 2020: 16th European Conference, Glasgow, UK, August 23-28, 2020, Proceedings, Part XIII 16, Springer International Publishing, 2020.

46 R. Ennis and K. Doerschner, The color appearance of curved transparent objects, J. Vis., 2021, 21(5), 20. 
47 M. Pharr, J. Wenzel, and G. Humphreys, Physically based rendering: From theory to implementation, Morgan Kaufmann, 2016.

48 Blender Manual, https://docs.blender.org/manual/en/latest/ render/shader_nodes/shader/principled.html.

$49 \mathrm{~K} . \mathrm{He}$, et al., Deep residual learning for image recognition." Proceedings of the IEEE conference on computer vision and pattern recognition, 2016.

50 L.-C. Chen, et al., Rethinking atrous convolution for semantic image segmentation, 2017, arXiv preprint arXiv:1706.05587.

51 J. Long, E. Shelhamer, and T. Darrell, Fully convolutional networks for semantic segmentation, Proceedings of the IEEE conference on computer vision and pattern recognition, 2015.

52 O. Ronneberger, P. Fischer, and T. Brox, U-net: Convolutional networks for biomedical image segmentation, International Conference on Medical image computing and computer-assisted intervention, Springer, Cham, 2015.

53 H. Fan, H. Su, and L. J. Guibas, A point set generation network for $3 \mathrm{~d}$ object reconstruction from a single image, Proceedings of the IEEE conference on computer vision and pattern recognition, 2017.
54 S. Eppel, and T. Kachman, Computer vision-based recognition of liquid surfaces and phase boundaries in transparent vessels, with emphasis on chemistry applications, 2014, arXiv preprint arXiv:1404.7174.

55 S. V. Ley, et al., Camera-enabled techniques for organic synthesis, Beilstein J. Org. Chem., 2013, 9(1), 1051-1072.

56 T. Zepel, et al., Automated liquid-level monitoring and control using computer vision, 2020.

57 G. Chen, K. Han, and K.-Y. K. Wong, Tom-net: Learning transparent object matting from a single image, Proceedings of the IEEE Conference on Computer Vision and Pattern Recognition, 2018.

58 W. Jang, et al., Structured-light stereo: Comparative analysis and integration of structured-light and active stereo for measuring dynamic shape, Opt. Lasers. Eng., 2013, 51(11), 1255-1264.

59 K. Rematas, R. Martin-Brualla, and V. Ferrari, Sharf: Shapeconditioned radiance fields from a single view, 2021, arXiv preprint arXiv:2102.08860.

60 NileRed Experimental chemistry youtube channel, https:// www.youtube.com/c/NileRed/videos.

61 J. Xiao, A. Owens, and A. Torralba, Sun3d: A database of big spaces reconstructed using sfm and object labels, Proceedings of the IEEE international conference on computer vision, 2013. 